\title{
Mechanical plus oral bowel preparation with paromomycin and metronidazole reduces infectious complications in elective colorectal surgery: a matched case-control study
}

\author{
Matthias Mehdorn ${ }^{1} \cdot$ Christoph Lübbert ${ }^{2,3} \cdot$ Iris F. Chaberny ${ }^{3,4} \cdot$ Ines Gockel ${ }^{1} \cdot$ Boris Jansen-Winkeln ${ }^{1}$ (I)
}

Accepted: 12 April 2021 / Published online: 25 April 2021

(C) The Author(s) 2021, corrected publication 2021

\begin{abstract}
Purpose Infectious complications are as high as $30 \%$ in elective colorectal surgery. In recent years, several studies have discussed the topic of preoperative bowel decontamination prior to colorectal surgery in order to reduce postoperative infectious complications and have found significant effects of oral antibiotic administration with a large variety of drugs used. No study has evaluated the combination of oral paromomycin and metronidazole in this context.

Methods We performed a prospective single-center study with a matched-pair retrospective cohort to evaluate postoperative infectious complications (superficial site infections, organ space abscess, anastomotic leakage) in elective colorectal surgery.

Patients A total of 120 patients were available for study inclusion; 101 gave informed consent and were included. A total of 92 patients were matched and subsequently analyzed. We could show a reduction in overall infectious complications in the intervention group $(15.2 \%$ vs $30.8 \%, p=0.018$; odds ratio $0.333,95 \%$ CI $0.142-0.784)$ as well as a reduction in superficial surgical site infections ( 8.7 vs $19.6 \%, p=0.041$, OR $0.333,95 \%$ CI $0.121-0.917$ ). The frequency of the other infectious complications such as intraabdominal abscesses and anastomotic leakage showed a tendency towards decreased frequencies in the intervention group (OR $0.714,95 \%$ CI $0.235-2.169$ and OR $0.571 ; 95 \%$ CI $0.167-1.952$, respectively). Finally, the oral antibiotic administration led to an almost significantly reduced length of stay (12.24 days vs 15.25 days; $p=0.057)$.

Conclusions Oral paromomycin and metronidazole with intravenous ertapenem effectively reduce infectious complications in elective colorectal surgery.

Trial registration The study was registered at Clinicaltrials.gov (NCT03759886) December 17, 2018
\end{abstract}

Keywords Surgical site infections - Antibiotic bowel preparation - Mechanic bowel preparation - Colorectal resections . Paromomycin

Boris Jansen-Winkeln

Boris.Jansen-Winkeln@medizin.uni-leipzig.de

1 Department of Visceral, Transplant, Thoracic and Vascular Surgery, Leipzig University Hospital, Liebigstr. 20,

D-04103 Leipzig, Germany

2 Division of Infectious Diseases and Tropical Medicine, Department of Medicine II, Leipzig University Hospital, Liebigstr. 20, D-04103 Leipzig, Germany

3 Interdisciplinary Center for Infectious Disease (ZINF), Leipzig University Hospital, Liebigstr. 20, 2204103 Leipzig, Germany

4 Institute of Hygiene, Hospital Epidemiology and Environmental Medicine, Leipzig University Hospital, Liebigstr, 22 04103 Leipzig, Germany

\section{Introduction}

Colorectal surgery is an operation prone to complications, in which up to $30 \%$ of all cases involve infectious complications such as anastomotic leakage, organ space abscesses, and superficial surgical site infections being present in up to $30 \%$ of all cases $[1,2]$.

In recent years, there has been an ongoing discussion over the past years about the benefits of oral antibiotics (OA) with or without mechanical bowel preparation (MBP) on infectious postoperative complications following colorectal resections. Over $90 \%$ of US colorectal surgeons apply mechanical as well as decontaminating bowel preparation [3]. Based on literature review, there have been recommendations to omit MBP [4] as an analysis of existing data in 2010 could not provide benefits on surgical site infections or anastomotic leakage in open 
colorectal surgery. Large-scale data analysis from the USA, taken from the ACS-NSQIP, has shown that MBP plus oral antibiotics prior to elective colorectal surgery was able to reduce overall surgical site infections by about $50 \%$ (14.7\% vs $6.2 \%$ ) [5]. Several other studies have based their analyses on this particular registry with different aspects highlighted, but all judged antibiotic preparation beneficial [5-12]. In general, a protective effect could be found in open and laparoscopic procedures [13]. A similar protective effect of oral antibiotic bowel preparation (OABP) without MBP could be shown in a meta-analysis that tried to point out the importance of OABP only for bowel preparation [14]. Recently, a large randomized controlled trial from Spain promoted the use of OABP without MBP to reduce SSIs in colorectal surgery [15].

In general, different antibiotic regimens are used in accordance with local availability of drugs and possible side effects. Most of the studies report the use of either an aminoglycoside (i.e., neomycine [16-18], kanamycine [19], streptomycin [20], gentamicine [21]) or a fluoroquinolone (levofloxacine [1] or ciprofloxacine [15]) plus metronidazole. Additionally, in all present studies, single-shot antibiotics were used preoperatively, mostly consisting of an IV application of a secondgeneration cephalosporine plus metronidazole intravenously. The ideal decontaminating agent remains in the bowel after oral ingestion. In Germany, most of the aminoglycosides used in other studies are only available as topic agents but not in an oral formulation designed for intestinal effects. An old but still common aminoglycoside is paromomycin which is used as a soluble powder approved for the prevention of hepatic encephalopathy and which is available in Germany. The only study that evaluated paromomycin for bowel decontamination in colorectal surgery is about 40 years old but already provided evidence for the possible use in colorectal surgery to reduce surgical site infections [22].

Since no data is available on the combined use of paromomycin and metronidazole for preoperative intestinal decontamination in colorectal surgery to prevent infectious complications, we conducted this prospective case-control study with matched-pair analysis.

The aim was to show a significant reduction of postoperative infectious complications by using paromomycin and metronidazole preoperatively after mechanical bowel preparation in colorectal surgery.

\section{Materials and methods}

\section{Patients and methods}

This clinical trial was implemented as a prospective, nonrandomized case-control study with matched-pair analysis of a retrospective cohort at the University Hospital Leipzig, Leipzig, Germany. Approved by the local ethics' committee of the Medical Faculty of the University of Leipzig (010/19ek), the study was registered at Clinicaltrials.gov (NCT03759886). Written informed consent was obtained from all patients involved. Inclusion criteria were all elective colorectal resections performed between January 2019 and January 2020, who could perform the assigned bowel preparation regime. Exclusion criteria were allergies against the used drugs, emergency surgery, obstructive bowel disease, and patient age under 18 years.

The study protocol for the intervention group (=IG) was as follows: during the afternoon of the day before surgery, patients had to drink 21 of MOVIPREP ${ }^{\circledR}$ solution as MBP (polyethyleneglycol; Norgine, Wettenberg, Germany) within a 2-h time frame. Directly after oral intake of MBP, all patients took 4-g paromomycin and 1-g metronidazole (MBP + OA) each as a single dose without any repetitive dosing. Additionally, all patients received a perioperative single shot for antibiotic prophylaxis with 1-g ertapenem IV within $60 \mathrm{~min}$ of skin incision. Due to the half-life of ertapenem, no second dose, even for longer operations, is needed.

After conclusion of the estimated study period in January 2020, we selected a retrospective matched cohort (control group (CG)) from the electronic patient charts with the same inclusion and exclusion criteria from our institution, who had undergone colorectal surgery between 1 January 2017 and 31 December 2018 to perform the statistical analysis to test for sufficient study power (see below). These patients underwent MBP with MOVIPREP $®$ only during the afternoon of the day before surgery. The perioperative single-shot antibiotic prophylaxis within $60 \mathrm{~min}$ from skin incision consisted of $1.5 \mathrm{-g}$ cefuroxime plus 500-mg metronidazole. A second dose of intravenous antibiotics was given $4 \mathrm{~h}$ after the initial dose if the duration of surgery was longer than $4 \mathrm{~h}$.

The following matching parameters were used with descending importance during the matching process to avoid inadequate matching: OPS code, diagnosis, age, BMI, and ASA score. For BMI, we accepted an age difference of \pm 5 $\mathrm{kg} / \mathrm{m}^{2}$, for age \pm 5 years. For ASA, we matched ASA III accordingly but ASA I or II interchangeably. If there was no matchable ASA III patient, comorbidities were evaluated for an equally comorbid ASA II patient as ASA is a nonetheless subjective parameter, set by the evaluating anesthesiologist.

All patients that had MBP + OA intake for more than 1 day prior to surgery or those refusing informed consent were excluded. The exact selection process is shown in Fig. 1.

In all study patients, we recorded the occurrence of superficial surgical site infections, organ space abscesses, anastomotic leakage, and their respective therapy in accordance with clinical grading $\mathrm{A}-\mathrm{C}$ [23] (reoperation $(\mathrm{C})$, radiologic drainage or endoscopic therapy (B), no therapy (A)) as well as postoperative paralytic ileus or infectious diarrhea. The evaluation of the SSI was performed by the treating surgeons. 


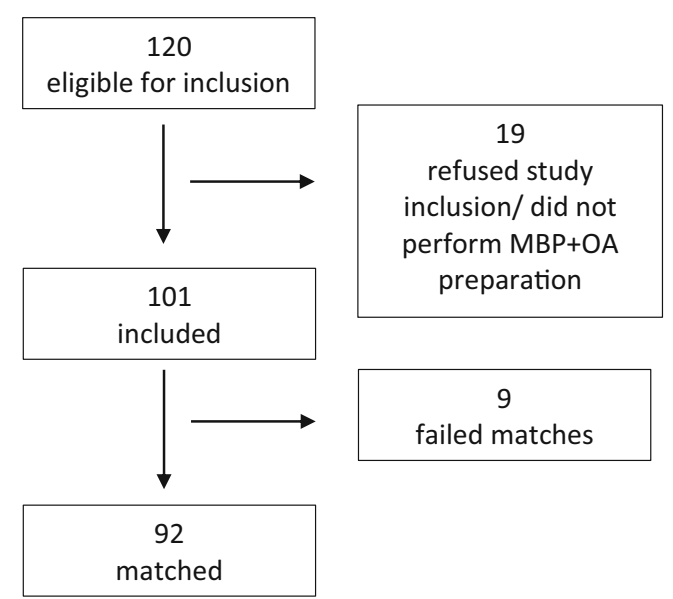

Fig. 1 The selection process

Follow-up was assured either by patients' visit in our institution or by a questionnaire that specifically asks for postoperative adverse events in the form of a check list (surgical site infection with specific treatment by their family doctor, surgery-related readmission) to evaluate for 30-day complication rates.

\section{Operative technique}

Our standards in colorectal surgery include a laparoscopic approach using a 4-incision laparoscopic or 6-incision robotic approach. For right hemicolectomy, a mini transverse laparotomy in the right upper quadrant was performed, and for left colonic, sigmoid, or rectal resections, the mini laparotomy in the lower left quadrant of approximately $6 \mathrm{~cm}$ is used. For wound edge protection, we use the "Alexis®" Wound Protector system (Applied Medical, Düsseldorf, Germany). For right hemicolectomy, an end-to-end anastomosis with PDS 4-0, extramucosal, continuous suture was performed extracorporeally. The distal resections are performed using the ECHELON FLEX $^{\mathrm{TM}}$ laparoscopic stapler and the ILS Circular Stapler (both Ethicon Endosurgery, Johnson \& Johnson, Norderstedt, Germany) for a circular stapled anastomosis. In lower rectal resections with total mesorectal excision (TME), we routinely sue diverting ileostomy, but not for partial mesorectal excision (PME). We use easy-flow drains in the vicinity of the anastomosis as indicator drains, which remain in place until the seventh day after surgery. Laparoscopic access sites are closed using intracutaneous sutures with Monocryl (Johnson \& Johnson, Norderstedt, Germany) or median laparotomies with staples. We do not place subcutaneous suction drains during wound closure. Peridural catheters or patient-controlled analgesia pumps are used according to patients' acceptance.

All procedures were performed by the senior author in association with another surgeon from the department. In 2017, we introduced the DaVinci robotic surgery platform to our department. Thus, an increasing number of rectal resections was performed robotically. No other differences in surgical technique or perioperative management occurred between both study periods except for the bowel preparation regime.

\section{Surgical site infections}

A superficial surgical site infection (SSSI) is considered if an operative wound shows signs of local inflammation (purulent drainage, swelling, erythema, local pain), a microorganism can be isolated from the wound fluid, or the wound is opened by the treating physician according to the American Center for Disease Control and Prevention (CDC). We assessed wounds daily during rounds to decide if the wound needs any further treatment. If so, we have a team of wound care specialists who are responsible for the treatment and if needed out-patient follow-up. Wounds are documented in a standard wound protocol that records the size of wounds and wound conditions, e.g., granulation and secretion. If the wound shows a small SSSI and no relevant amount of purulent secretion occurs, no wound swap is taken as it does not influence local therapy. If a local phlegmon is present and the wound discharges purulent fluids, wound swaps are taken for bacterial culture analysis in order to be able to administer targeted antibiotic therapy, but we did not perform routine swaps for study purposes.

Elevated inflammatory parameters, abdominal pain, or other signs of systemic inflammation raise the suspicion of an intraabdominal complication such as intraabdominal abscess or anastomotic leakage (AL). Patients routinely receive CT scans for further evaluation. If there is an intraabdominal abscess, depending on its size and approachability for radiologyguided drain placement, patients receive the appropriate treatment. Only postoperative intraabdominal abscesses that needed further treatment were recorded in the respective complication category. Usually, fluid from abscesses with drain placement is sent for bacterial culture. Patients with rectal resections receive a routine rectoscopy in our department after 7 days to check the anastomosis. As we routinely place a diverting loop ileostomy, endoscopic sponge treatment is initiated, if an AL grade B occurs.

\section{External validation of study results}

After completion of the study analysis, we compared our study results to the external quality control by the German National Reference Center for the Surveillance of Nosocomial Infections (www.nrz-hygiene.de). Here, specific operations, i.e., colorectal resections, are selected according to the OPS code (emergency and elective surgeries). Our own data is collected and validated by our Institute of Hygiene, Hospital Epidemiology, and Environmental Medicine of the Leipzig University Hospital within a follow-up period of 30 days. The data of all treated patients was evaluated 
independently of their study inclusion. In parallel, the general treatment standard in colorectal surgery was amended according to the study protocol. Thus, the quality control data show the change in nosocomial surgical site infections before and after the start of our study as real-world data. The wound infection rate (WIR) is defined as the number of wound infections in the indicator procedure divided by the count of all indicator procedures multiplied by 100 .

\section{Power calculation}

We estimated a case load of approximately 120 patients eligible for study inclusion per year. From the abovementioned literature $[1,24,25]$, an odds ratio of 2 to 3 could be expected for the reduction of infectious complications. Subsequently, the number of corresponding pairs would be between 72 and 110 to reach a power of 0.8 with the $a$-error set at 0.05 . The power calculation was performed using the open-source software G*power (Düsseldorf, Germany) with assistance from the local Institute for Medical Computer Science, Statistics, and Epidemiology (IMISE).

\section{Data collection and analysis}

Data were collected from the electronic patient charts in the same way for the retrospective and prospective cohort using MS Excel 2016 (Microsoft Corp., Munich; Germany) and analyzed using SPSS 24 (IBM, Ehningen, Germany). For comparison of continuous variables, we used the paired $t$-test, and for discrete variables the McNemar test. For the calculation of the odds ratio (OR) and its respective $95 \%$ confidence levels $(95 \% \mathrm{CI})$, we used the conditional logistic regression. The significance level was set to be $p=0.05$.

\section{Results}

Between 1 January 2019 and 31 January 2020, a total of 120 patients were found eligible for inclusion. Of those, 19 had to be excluded for various reasons (i.e., no OA received, refusal of informed consent, inability to consent, postponement of surgery). Thus, 101 patients (71 males, 30 females) were included in the study and completed the study protocol. After the completion of the trial period, we performed the matching process in accordance with the before-mentioned parameters. After the primary analysis of the main outcome parameter, i.e., reduction in SSI, we concluded further inclusion of patients as power levels were reached. A total of 92 of those 101 patients were matchable, excluding 5 male and 4 female patients because of the set matching variables' limits (Fig. 1). The patient characteristics of our intervention group (IG) and the matched control group (CG) are listed in Table 1. The matching process resulted in equally distributed comorbidities.
Most common indications for surgery were colon and rectal cancer as well as sigmoid diverticulitis. This is reflected in the resections performed, with rectal resection $(35.1 \%)$, sigmoid resection/left colectomy (35.1\%), and right colectomy (25\%) being the most common interventions (Table 2).

In the IG, the overall rate of total infections $(15.2 \%$ vs $30.4 \%, p=0.013$ ) was significantly reduced with an odds ratio (OR) of 0.333 (95\% CI 0.142-0.784; power 0.86) (Table 3). The incidence of superficial SSI ( 8.7 vs $19.6 \%, p=0.041)$ was significantly lower in the IG with an OR of $0.333(95 \%$ CI $0.121-0.917$, power 0.74$)$. The CG had predominantly superficial SSI involving the abdominal laparotomy wound ( $p=$ 0.049). Nonetheless, wound measures did not differ between both groups. With regard to the surgical approach, preoperative bowel decontamination showed a significant decrease in SSI in open surgery $(p=0.018)$, but a less remarkable effect in laparoscopic surgery $(p=0.263)$.

Intraabdominal abscesses (OR $0.714,95 \%$ CI 0.235 2.169) or anastomotic leakage (OR $0.571 ; 95 \%$ CI $0.167-$ 1.952) showed a trend towards a decreased frequency in the IG, but did not reach a significant level. The treatment of anastomotic leakage differed between both groups as only one patient in the IG but five patients in the CG had grade C $\operatorname{AL}(p=0.287)$.

Patients with oral bowel preparation with $\mathrm{MBP}+\mathrm{OA}$ showed a decrease in postoperative paralytic ileus requiring prokinetic medication or nasogastric tube placement, but which also did not reach significant levels (10.9\% vs $17.4 \%$; $p=0.26$; OR $0.538,95 \%$ CI $0.215-1.350$ ).

All other postoperative complications were similar in both groups except for cardioembolic events of which 3 occurred in the IG.

The treatment with preoperative bowel decontamination indicated a shorter hospital stay (LOS) in the IG of about 3 days (12.24 vs 15.25 days; $p=0.057$ ).

There was no difference regarding Clostridioides difficileassociated diarrhea (CDAD) between IG and CG. No other adverse events were reported with regard to paromomycin or metronidazole application.

\section{Bacterial and fungal isolates}

In the preoperative screening tests for multidrug-resistant organisms (MDRO), we found seven patients being colonized with MDR Escherichia coli in the IG compared to eight patients in the CG. In the CG, we also found methicillin-resistant Staphylococcus aureus (MRSA) strains, vancomycinresistant enterococci (VRE, Enterococcus faecium), and a MDR Enterobacter hormaechei strain (Table 4). As we only collected wound specimens from one wound for microbiological assessment in the IG, we only detected a Streptococcus agalactiae strain. In the CG, a wide variety of mostly gramnegative rods and enterococci were detected, with three of 
Table 1 Patient characteristics. Values given as mean $( \pm$ standard deviation) or absolute number (frequency). $B M I$ body mass index in $\mathrm{kg} / \mathrm{m}^{2}, C A D$ coronary artery disease, $C H F$ congestive heart failure, ASA American Society of Anesthesiologists performance score

\begin{tabular}{llll}
\hline Patient characteristics & Intervention group & Control group & $p$-value \\
\hline Age & $63.17( \pm 12.8)$ & $62.68( \pm 12.1)$ & 0.748 \\
Sex (male/female) & $66 / 26$ & $66 / 26$ & \\
BMI & $26.04( \pm 4.9)$ & $26.1( \pm 4.2)$ & 0.913 \\
Comorbidities & & & \\
Hypertension & $56(60.9)$ & $58(63)$ & 0.864 \\
Peripheral artery disease & $3(3.3)$ & $3(3.3)$ & 1 \\
CAD/CHF & $19(20.7)$ & $19(20.7)$ & 1 \\
Diabetes & $18(19.7)$ & $16(17.4)$ & 0.839 \\
Liver cirrhosis & $3(3.3)$ & $2(2.2)$ & 1 \\
Malignant disease & $70(76.1)$ & $63(68.5)$ & 0.118 \\
Chemotherapy & $30(32.6)$ & $26(28.3)$ & 0.503 \\
Immunosuppression & $7(7.6)$ & $4(4.3)$ & 0.508 \\
Chronic inflammatory disease & $6(6.5)$ & $5(5.4)$ & 1 \\
Renal insufficiency & $39(42.2)$ & $45(48.9)$ & 0.392 \\
Colonization with multidrug-resistant bacteria & $7(7.6)$ & $13(14,1)$ & 0.263 \\
Preoperative albumin in mg/dl & $43.15( \pm 4.42)$ & $42.59( \pm 4.67)$ & 0.378 \\
ASA score & & & \\
I & $1(1.1)$ & $7(7.6)$ & $0.046^{*}$ \\
II & $65(70.7)$ & $53(57.6)$ & \\
III & $26(28.3)$ & $31(33.7)$ & \\
\hline
\end{tabular}

them being colonized with Candida species. One versus three patients had MDRO in their wound fluid cultures in the IG and the $\mathrm{CG}$, respectively.

The results of our own hospital infection surveillance system, which is monitored by the German National Reference Center, show high nosocomial infection rates prior to the start of our study: $8.51 \%$ and $38.89 \%$ in the laparoscopic and the open surgery group respectively (see Fig. 2). Since the introduction of our MBP+OA standard, the WIR in open surgery has decreased to $8.2 \%$ in open and $5.88 \%$ in laparoscopic colon resection.

\section{Discussion}

In this prospective matched case-control study, we sought to evaluate the effects of preoperatively administered oral paromomycin and metronidazole together with mechanical bowel preparation and IV ertapenem to reduce infectious complications in elective colorectal surgery.

Recent literature on preoperative oral antibiotic bowel preparation (OABP) heavily relies on results taken from the ACSNSQIP registry for different colorectal procedures [5-12]. These studies prefer OABP + MBP as protective regimen with regard to infectious postoperative complications. The recent meta-analysis carried out by Mulder et al. [14] excluded most of the ACS-NSQIP studies to prevent bias by multiple inclusions of the same patients. Nonetheless, they found a benefit of preoperative OABP. Those registry data only take into account if patients received any kind of antibiotic bowel preparation without paying giving any further detail in the specific regimen given. Unfortunately, those data only promote OABP but do not show any superior regimen so that colorectal surgeons cannot use those data as a proper guideline. Furthermore, OABP without MBP has to be taken into consideration, as recent evidence by the ORALEV trial exists, that the mechanical preparation maybe does not exert any additional benefits in reducing the postoperative infectious complications $[14,15]$.

Internationally, a wide variety of antibiotic regimen can be found. The common combination of an orally available aminoglycoside along with metronidazole, beginning 1 or 2 days preoperatively, is influenced by local drug legislation. For example, several Asian studies report the use of kanamycine $[19,26]$, whereas the Angloamerican studies mostly have used neomycine $[27,28]$. In Germany, most of the studied aminoglycosides do not exist as orally available drug formulations. We therefore chose paromomycin as an orally available aminoglycoside, which has been proven to reduce infectious complications in colorectal surgery used in bowel irrigation preoperatively [22]. Nonetheless, data is scarce on its potential in combination with metronidazole, which is a common adjunct antibiotic in bowel preparation. Metronidazole is an antibiotic drug that especially is effective against anaerobic bacteria such as Bacteroides spp. and Clostridoides spp., common intestinal bacteria that are isolated from SSI [29]. In our intervention group (IG), we found an OR of 0.333 for total and 
Table 2 Indications for surgery and the respective procedures.

Values are given as mean $( \pm$ standard deviation) or absolute number (frequency). $C D C$ US Center for Disease Control and Prevention

\begin{tabular}{|c|c|c|c|}
\hline & Intervention group & Control group & $p$-value \\
\hline \multicolumn{4}{|l|}{ Indications } \\
\hline Colon cancer & $16(17.4)$ & $19(20.7)$ & \\
\hline Sigmoid cancer & $9(9.8)$ & $8(8.7)$ & \\
\hline Rectal cancer & $29(31.5)$ & $27(29.3)$ & \\
\hline Sigmoid diverticulitis & $23(25)$ & $25(29.3)$ & \\
\hline Polyps of colon & $6(6.5)$ & $4(4.3)$ & \\
\hline Inflammatory bowel disease & $2(2.2)$ & $3(3.3)$ & \\
\hline Peritoneal pseudomyxoma & $2(2.2)$ & $2(2.2)$ & \\
\hline Radiogenic rectal stenosis & $1(1.1)$ & 0 & \\
\hline Sigmoid vesical fistula & $2(2.2)$ & 0 & \\
\hline Anal carcinoma & $1(1.1)$ & $2(2.2)$ & \\
\hline Others & $2(2.2)$ & $2(2.2)$ & \\
\hline \multicolumn{4}{|l|}{ Resections } \\
\hline Right colectomy & $23(25 \%)$ & $23(25 \%)$ & \\
\hline Left colectomy & $2(2.2)$ & $2(2.2)$ & \\
\hline Left colectomy/sigmoid & $30(32.6)$ & $30(32.6)$ & \\
\hline Rectal resection & $25(27.5)$ & $25(27.5)$ & \\
\hline Proctocolectomy & $2(2.2)$ & $2(2.2)$ & \\
\hline Hartmann's reversal & $2(2.2)$ & $2(2.2)$ & \\
\hline Extralevatoric rectal exstirpation & $5(5.4)$ & $5(5.4)$ & \\
\hline Proctocolectomy with pouch & $1(1.1)$ & $1(1.1)$ & \\
\hline Rectal resection with colostomy & $2(2.2)$ & $2(2.2)$ & \\
\hline \multicolumn{4}{|l|}{ Surgical approach } \\
\hline Laparoscopic & $79(85.9)$ & $79(85.9)$ & \\
\hline Open & $13(14.1)$ & $13(14.1)$ & \\
\hline Duration of surgery & $243.95( \pm 106.4)$ & $247.21( \pm 122.8)$ & 0.739 \\
\hline \multicolumn{4}{|l|}{ Wound contamination class (CDC) } \\
\hline Clean-contaminated (II) & $84(91.3)$ & 79 (85.9) & 0.246 \\
\hline Contaminated (III) & $8(8.3)$ & $13(14.1)$ & \\
\hline
\end{tabular}

superficial surgical site infections when taking MBP + OA, which is a similar effect compared to the study by Abis et al. [30], but superior to the results demonstrated by Mulder et al. $[25,31]$. Abis et al. performed a proper selective intestinal decontamination by prolonged perioperative administration of the drugs for at least 6 days. Both groups used tobramycin and colistin for 1 and 3 days respectively, but Abis et al. also added amphotericin $\mathrm{B}$ as an anti-fungal agent. This raises the question of the need for additional anti-fungal bowel preparation. We found some yeast species in the swaps of the CG but none in the IG. This is most likely due to the small number of swaps taken in the IG. At least all fungi (Candida albicans and Candida krusei) would have been sensitive to amphotericin B. Unfortunately, the studies using amphotericin B as an oral decontamination agent do not describe wound cultures in order to justify the necessity of addition of anti-fungal agents.

We used ertapenem as a single-shot perioperative IV antibiotic. Other studies routinely used cephalosporines combined with metronidazole. We purposefully chose this first- generation carbapenem to reach a better coverage of the intestinal microbiota including extended-spectrum beta-lactamases (ESBL)-producing enterobacteria combined with the longer plasma half-life of ertapenem of about $4 \mathrm{~h}$ [32]. Additionally, ertapenem is effective against most of the gram-negative bacteria that often cause SSI in colorectal surgery (i.e., Escherichia coli, Klebsiella pneumonia), gram-positive anaerobes, and gram-positive cocci such as Streptococcus pneumoniae [29]. The regime of cefuroxime and metronidazole is less effective against those gram-positive and gramnegative anaerobes as well as enterobacterales according to the most recent EUCAST report [33]. Even some Enterococcus faecalis strains are susceptible to ertapenem. The only available study comparing different single-shot antibiotic regimen in colorectal surgery found a slightly higher SSI rate using ertapenem compared to cefazoline plus metronidazole (OR 1.48, not significant) [34]. Overall, they attributed the greatest effect in SSI prevention to the oral decontamination and not to IV single-shots. By using ertapenem instead 
Table 3 Postoperative general and infectious complications. SSI surgical site infection, $C D A D C$. difficile-associated diarrhea, AKIN acute kidney injury

\begin{tabular}{|c|c|c|c|c|c|}
\hline & Intervention group & Control group & $p$-value & Odds ratio & $95 \% \mathrm{CI}$ \\
\hline \multicolumn{6}{|l|}{ Infectious complications } \\
\hline Total infections & $14(15.2)$ & $28(30.4)$ & $0.013 *$ & 0.333 & $0.142-0.784$ \\
\hline Superficial SSI & $8(8.7)$ & $18(19.6)$ & $0.041 *$ & 0.333 & $0.121-0.917$ \\
\hline Abdominal & $5(5.4)$ & $14(15.2)$ & $0.049 *$ & & \\
\hline Perineal & $1(1.1)$ & $2(2.2)$ & & & \\
\hline Anal suture & $2(2.2)$ & 0 & & & \\
\hline \multicolumn{6}{|l|}{$\begin{array}{l}\text { Wounds according to } \\
\text { surgical approach }\end{array}$} \\
\hline Laparoscopic & $5(6.3)$ & $9(11.4)$ & 0.263 & & \\
\hline Open & $3(23)$ & $9(70)$ & $0.018 *$ & & \\
\hline Anastomotic leakage[23] & $5(6.1)$ & $9(11)$ & 0.549 & 0.571 & $0.167-1.952$ \\
\hline Grade A & $1(1.1)$ & $1(1.1)$ & 0.287 & & \\
\hline Grade B & $3(3.3)$ & $3(3.3)$ & & & \\
\hline Grade C & $1(1.1)$ & $5(5.5)$ & & & \\
\hline Intraabdominal abscess & $5(5.4)$ & $7(7.6)$ & 0.754 & 0.714 & $0.235-2.169$ \\
\hline \multicolumn{6}{|l|}{ General complications } \\
\hline Paralysis & $10(10.9)$ & $16(17.4)$ & 0.26 & 0.538 & $0.215-1.350$ \\
\hline Diarrhea & $5(5.4)$ & $5(5.4)$ & 1 & & \\
\hline CDAD & $1(1.1)$ & $2(2.2)$ & 1 & & \\
\hline Pneumonia & $3(3.3)$ & $2(2.2)$ & 1 & & \\
\hline Bleeding & $4(4.3)$ & $1(1.1)$ & 0.375 & & \\
\hline Delirium & $0(0)$ & $0(0)$ & 1 & & \\
\hline Cardioembolic & $3(3.3)$ & 0 & 0.25 & & \\
\hline AKIN & $12(13)$ & $15(16.3)$ & 0.648 & & \\
\hline Death & $2(2.2)$ & $1(1.1)$ & 1 & & \\
\hline Readmission due to SSI & $3(3.3)$ & $5(5.4)$ & 0.688 & 0.500 & $0.092-2.730$ \\
\hline Postoperative length of stay & $12.24( \pm 10.3)$ & $15.25( \pm 12.51)$ & 0.057 & & \\
\hline
\end{tabular}

Significant $(\mathrm{p}<0.05) \mathrm{p}$-values are marked with * of cefotaxime preoperatively, we could cover all resistant gram-negative bacteria, which we had identified preoperatively by routine screenings. Unfortunately, ertapenem does not cover Pseudomonas aeruginosa which may be responsible for AL development under certain circumstances [35]. Still, it seems impossible to cover any possible bacteria with a single-shot antibiotic with the intention to prevent SSI, especially since some of the data on Pseudomonas aeruginosa and Enterococcus faecalis and their role on anastomotic leakage are in vitro experiments $[35,36]$. After all, you have to keep in mind that a responsible administration of reserve antibiotics to prevent bacterial resistance generation is mandatory, and therefore, clinical evaluation of bacterial prevalences in SSI is necessary.

The most pronounced effect of preoperative bowel preparation could be seen in the open surgical group, which was by far the minority of surgical procedures (14\%). The respective infection rates were $70 \%$ compared to $23 \%$ in the laparoscopic surgery group. This is in line with common knowledge on the advantages of laparoscopic surgery. Nevertheless, our results reflect those of Özdemir et al. [21] who evaluated OABP (gentamycine and metronidazole) in open colorectal surgery. They also demonstrated a decrease in SSI from 71 to $36 \%$ by using OABP and calculated subsequent savings of about roughly 1700 USD per patient per hospital stay. Similarly, Suzuki et al. [19] found the majority of infections in the open surgical subgroup of their cohort of both open and laparoscopic colorectal cancer surgeries, but they attributed the reduction in infection rates to the addition of mechanical bowel preparation. The SSI rates we found in the CG are relatively high but still in the range of the aforementioned studies. But this elevated rate of SSI stresses the need for measures to improve postoperative patient outcome with regard to infectious complications.

Reduction of anastomotic leakage (AL) has been considered a primary end point in several studies that evaluated preoperative bowel decontamination. Schardey et al. [37] terminated their study after an interim analysis because of a significant reduction in AL in rectal resections. They used a very broad combination of topical antibiotic agents comprising tobramycin, vancomycin, polymyxin B, and amphotericin B 
Table 4 Multidrug-resistant (MDR) bacteria identified by preoperative screening and results of postoperative microbiological cultures. MRSA methicillin-resistant Staphylococcus aureus, VRE vancomycin-resistant Enterococcus

\begin{tabular}{lll}
\hline & Intervention group & Control group \\
\hline Preoperative MDR bacteria & & \\
MDR Escherichia coli & $7(100)$ & $8(75)$ \\
MRSA & 0 & $2(16.7)$ \\
VRE Enterococcus faecium & 0 & $2(16.7)$ \\
MDR Enterobacter hormachaei & 0 & $1(8.3)$ \\
Swaps taken & $1(1.1)$ & $12(13.4)$ \\
Swaps sterile & 0 & $1(8.3)$ \\
MDR in wound & 1 & 3 \\
Bacterial isolates & & \\
Staph. epidermidis & 0 & $1(8.3)$ \\
Escherichia coli & 0 & $5(41.7)$ \\
Enterococcus faecium & $1(100)$ & $1(8.3)$ \\
Enterococcus faecalis & 0 & $2(16.7)$ \\
Pseudomonas aeruginosa & 0 & $3(25)$ \\
Morganella morgagni & 0 & $1(8.3)$ \\
Candida albicans & 0 & $2(16.7)$ \\
Proteus mirabilis & 0 & $1(8.3)$ \\
Eenterobacter aerogenes & 0 & $1(8.3)$ \\
Candida krusei & 0 & $1(8.3)$ \\
Streptococcus agalactiae & $1(100)$ & 0 \\
\hline & &
\end{tabular}

versus placebo plus amphotericin B. Their intention was to double cover gram-positive microorganisms after elimination of gram-negative flora. Another randomized study to assess anastomotic leakage in colorectal surgery was recently carried out in the Netherlands [31]. The study was terminated prematurely, as an OR of 0.8 for deep SSI and 0.5 for anastomotic leakage was found. At the point of study cessation, they had included 80 of 966 estimated patients and found 1 versus 2 anastomotic leakages. Hence, it seems doubtful if the authors' calculated ORs really represent a statistical effect that justifies the termination of the study. Other data hints at reduced $\mathrm{AL}$ rates after MBP + OA. Even less aggressive bowel preparation regime (neomycine and metronidazole) could show a reduction in AL in a very recent randomized trial [38]. But almost simultaneously, a different study group published contradictory data with no reduction in AL but slightly lower rates of SSI [39]. We could show a reduced AL rate with less severe $\mathrm{AL}$ grades in the IG which did not reach significance levels. The necessary treatment differed remarkably with 5 relaparotomies in the CG versus 1 in the IG, but AL treatment overall did not reach significant levels $(p=0.287)$. This difference is due to the different localization of anastomosis, i.e., rectal versus colonic anastomosis. The detected trend towards a shorter LOS in the IG might also be attributed to the higher rate of reoperation. Although, conservative treatment of an $\mathrm{AL}$ (grades A and B) with endoscopic sponge therapy might be even more time-consuming until a sufficient granulation of anastomosis is assured. In total, 10 procedures per group were performed without an anastomosis reducing the subgroup for the analysis of AL. Therefore, a greater cohort is needed to demonstrate a statistically significant effect of our bowel preparation regime with regard to AL. Still, our data taken together
Fig. 2 Number of SSI per 100 indicator procedures

\section{Number of SSI per 100 indicator procedures}

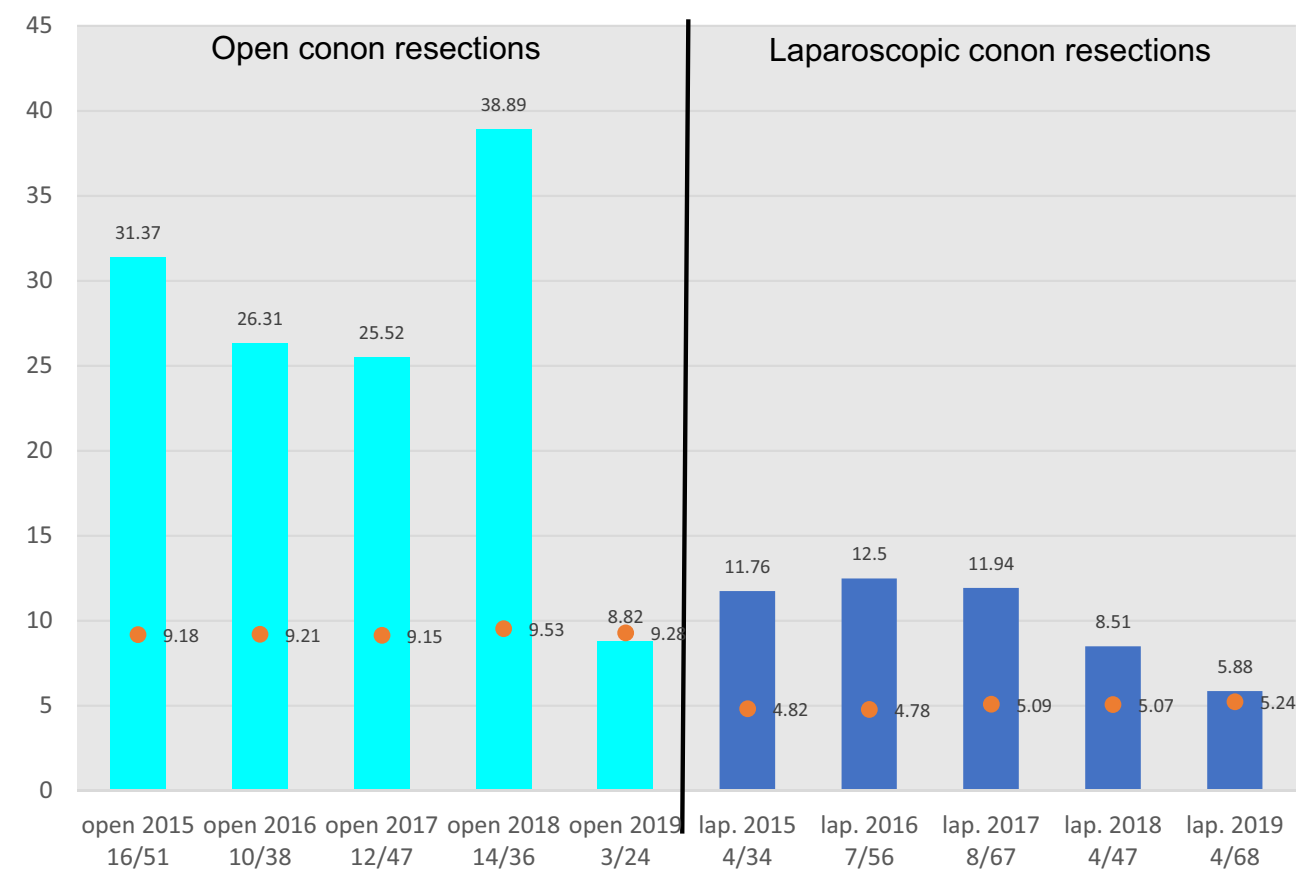


with existing data provides diverse recommendations with regard to $\mathrm{AL}$ reduction which further stresses the necessity of studies on the best antibiotic regime for perioperative bowel preparation.

Regardless of the statistically significant changes due to the introduction of OA with MBP + OA discovered in our study, the external validation using the national surveillance data of our institution confirms our observations and stresses the importance of MBP + OA.

Our study has several limitations. First and foremost, we conducted a prospective study with matching of a historical control group. The matching process was performed by the authors manually in a blinded fashion from a patient list maintaining the matching variables only. Due to the manual selection pattern, a possible matching bias cannot be excluded. The inclusion of several rare procedures such as proctocolectomy or Hartmann's reversal might influence the results as well. However, since those patients were properly matched, we do not assume great effects, especially since those procedures consist of the main surgical steps as the more common procedures (i.e., surgical approach, extracorporeal bowel resection, transanal anastomosis, placement of ostomy).

As several studies hint at the benefits of OABP in the prevention of infectious complications in colorectal surgery, future studies should focus on the best antibiotic regimen for preoperative bowel decontamination depending on the microbiological background epidemiology. To date, the inhomogeneity of the regimens used with respect to antimicrobials and drug dosages is still a major problem in the field of OABP.

\section{Conclusion}

Based on the results of our matched-pair analysis, we propose oral paromomycin and metronidazole in single doses for oral antibiotic bowel preparation, paired with a single shot of IV ertapenem preoperatively in colorectal surgery as a promising option to effectively reduce postoperative infectious complications.

Acknowledgements We thank Silke Zachariae (IMISE) for the statistical advice and support during study planning and analysis.

Availability of data and material

The data is available from the corresponding author upon reasonable request.

Funding Open Access funding enabled and organized by Projekt DEAL.

\section{Declarations}

Ethics approval The study was approved by the ethics committee of the Medical Faculty of the University of Leipzig (010/19-ek).
Consent to participate All patients of the intervention group gave written informed consent to participate in the study. Consent of the retrospectively matched cohort was waived by the ethics committee.

Conflict of interest The authors declare no competing interests.

Open Access This article is licensed under a Creative Commons Attribution 4.0 International License, which permits use, sharing, adaptation, distribution and reproduction in any medium or format, as long as you give appropriate credit to the original author(s) and the source, provide a link to the Creative Commons licence, and indicate if changes were made. The images or other third party material in this article are included in the article's Creative Commons licence, unless indicated otherwise in a credit line to the material. If material is not included in the article's Creative Commons licence and your intended use is not permitted by statutory regulation or exceeds the permitted use, you will need to obtain permission directly from the copyright holder. To view a copy of this licence, visit http://creativecommons.org/licenses/by/4.0/.

\section{References}

1. Anjum N, Ren J, Wang G, Li G, Wu X, Dong H, Wu Q, Li J (2017) A randomized control trial of preoperative oral antibiotics as adjunct therapy to systemic antibiotics for preventing surgical site infection in clean contaminated, contaminated, and dirty type of colorectal surgeries. Dis Colon Rectum 60(12):1291-1298. https://doi.org/10.1097/dcr.0000000000000927

2. Roos D, Dijksman LM, Oudemans-van Straaten HM, de Wit LT, Gouma DJ, Gerhards MF (2011) Randomized clinical trial of perioperative selective decontamination of the digestive tract versus placebo in elective gastrointestinal surgery. Br J Surg 98(10): 1365-1372. https://doi.org/10.1002/bjs.7631

3. McChesney SL, Zelhart MD, Green RL, Nichols RL (2020) Current U.S. pre-operative bowel preparation trends: a 2018 survey of the American Society of Colon and Rectal Surgeons Members. Surg Infect 21(1):1-8. https://doi.org/10.1089/sur.2019.125

4. Eskicioglu C, Forbes SS, Fenech DS, McLeod RS (2010) Preoperative bowel preparation for patients undergoing elective colorectal surgery: a clinical practice guideline endorsed by the Canadian Society of Colon and Rectal Surgeons. Can J Surg 53(6):385-395

5. Kiran RP, Murray ACA, Chiuzan C, Estrada D, Forde K (2015) Combined preoperative mechanical bowel preparation with oral antibiotics significantly reduces surgical site infection, anastomotic leak, and ileus after colorectal surgery. Ann Surg 262(3):416-425; discussion 23-5. https://doi.org/10.1097/sla.0000000000001416

6. Al-Mazrou AM, Hyde LZ, Suradkar K, Kiran RP (2018) Effect of inclusion of oral antibiotics with mechanical bowel preparation on the risk of Clostridium difficile infection after colectomy. Journal of gastrointestinal surgery : official journal of the Society for Surgery of the Alimentary Tract 22(11):1968-1975. https://doi.org/10. 1007/s11605-018-3837-3

7. Garfinkle R, Abou-Khalil J, Morin N, Ghitulescu G, Vasilevsky CA, Gordon P, Demian M, Boutros M (2017) Is there a role for oral antibiotic preparation alone before colorectal surgery? ACS-NSQIP analysis by coarsened exact matching. Dis Colon Rectum 60(7): 729-737. https://doi.org/10.1097/dcr.0000000000000851

8. Kaslow SR, Gani F, Alshaikh HN, Canner JK (2018) Clinical outcomes following mechanical plus oral antibiotic bowel preparation versus oral antibiotics alone in patients undergoing colorectal surgery. BJS open 2(4):238-245. https://doi.org/10.1002/bjs5.66 
9. Klinger AL, Green H, Monlezun DJ, Beck D, Kann B, Vargas HD, Whitlow C, Margolin D (2019) The role of bowel preparation in colorectal surgery: results of the 2012-2015 ACS-NSQIP Data. Ann Surg 269(4):671-677. https://doi.org/10.1097/sla. 0000000000002568

10. Morris MS, Graham LA, Chu DI, Cannon JA, Hawn MT (2015) Oral antibiotic bowel preparation significantly reduces surgical site infection rates and readmission rates in elective colorectal surgery. Ann Surg 261(6):1034-1040. https://doi.org/10.1097/sla. 0000000000001125

11. Parthasarathy M, Bowers D, Groot-Wassink T (2018) Do preoperative oral antibiotics increase Clostridium difficile infection rates? An analysis of 13959 colectomy patients. Colorectal disease: the official journal of the Association of Coloproctology of Great Britain and Ireland 20(6):520-528. https://doi.org/10.1111/codi. 13926

12. Scarborough JE, Mantyh CR, Sun Z, Migaly J (2015) Combined mechanical and oral antibiotic bowel preparation reduces incisional surgical site infection and anastomotic leak rates after elective colorectal resection: an analysis of colectomy-targeted ACS NSQIP. Ann Surg 262(2):331-337. https://doi.org/10.1097/sla. 0000000000001041

13. Luo J, Liu Z, Pei KY, Khan SA, Wang X, Yang M, Wang X, Zhang $Y$ (2019) The role of bowel preparation in open, minimally invasive, and converted-to-open colectomy. J Surg Res 242:183-192. https://doi.org/10.1016/j.jss.2019.02.039

14. Mulder T, Kluytmans J (2019) Oral antibiotics prior to colorectal surgery: do they have to be combined with mechanical bowel preparation? Infect Control Hosp Epidemiol 40(8):922-927. https://doi. org/10.1017/ice.2019.157

15. Espin Basany E, Solís-Peña A, Pellino G, Kreisler E, Fraccalvieri D, Muinelo-Lorenzo M, Maseda-Díaz O, García-González JM, Santamaría-Olabarrieta M, Codina-Cazador A, Biondo S (2020) Preoperative oral antibiotics and surgical-site infections in colon surgery (ORALEV): a multicentre, single-blind, pragmatic, randomised controlled trial. Lancet Gastroenterol Hepatol 5:729738. https://doi.org/10.1016/s2468-1253(20)30075-3

16. Golder AM, Steele CW, Conn D, MacKay GJ, McMillan DC, Horgan PG, Roxburgh CS, McSorley ST (2019) Effect of preoperative oral antibiotics in combination with mechanical bowel preparation on inflammatory response and short-term outcomes following left-sided colonic and rectal resections. BJS open 3(6):830-839. https://doi.org/10.1002/bjs5.50224

17. Vadhwana B, Pouzi A, Surjus Kaneta G, Reid V, Claxton D, Pyne L, Chalmers R, Malik A, Bowers D, Groot-Wassink T (2020) Preoperative oral antibiotic bowel preparation in elective resectional colorectal surgery reduces rates of surgical site infections: a single-centre experience with a cost-effectiveness analysis. Ann R Coll Surg Engl 102(2):133-140. https://doi.org/10.1308/ rcsann.2019.0117

18. Xiaolong X, Yang W, Xiaofeng Z, Qi W, Bo X (2018) Combination of oral nonabsorbable and intravenous antibiotics versus intravenous antibiotics alone in the prevention of surgical site infections after elective colorectal surgery in pediatric patients: a retrospective study. Medicine. 97(36):e12288. https://doi.org/10. 1097/md.0000000000012288

19. Suzuki T, Sadahiro S, Tanaka A, Okada K, Saito G, Miyakita H, Ogimi T (2019) Usefulness of preoperative mechanical bowel preparation in patients with colon cancer who undergo elective surgery: a prospective randomized trial using oral antibiotics. Digestive surgery:1-7. https://doi.org/10.1159/000500020

20. Lei P, Ruan Y, Yang X, Wu J, Hou Y, Wei H, Chen T (2020) Preoperative mechanical bowel preparation with oral antibiotics reduces surgical site infection after elective colorectal surgery for malignancies: results of a propensity matching analysis. World $\mathrm{J}$ Surg Oncol 18(1):35. https://doi.org/10.1186/s12957-020-1804-4
21. Ozdemir S, Gulpinar K, Ozis SE, Sahli Z, Kesikli SA, Korkmaz A, Gecim IE (2016) The effects of preoperative oral antibiotic use on the development of surgical site infection after elective colorectal resections: a retrospective cohort analysis in consecutively operated 90 patients. Int J Surg 33(Pt A):102-108. https://doi.org/10.1016/j. ijsu.2016.07.060

22. Jostarndt L, Thiede A, Sonntag HG, Hamelmann H (1981) Systemic antibiotic prophylaxis in elective colon surgery. Results of a controlled study. Chirurg. 52(6):398-402

23. Rahbari NN, Weitz J, Hohenberger W, Heald RJ, Moran B, Ulrich A, Holm T, Wong WD, Tiret E, Moriya Y, Laurberg S, den Dulk M, van de Velde C, Buchler MW (2010) Definition and grading of anastomotic leakage following anterior resection of the rectum: a proposal by the International Study Group of Rectal Cancer. Surgery. 147(3):339-351. https://doi.org/10.1016/j.surg.2009.10. 012

24. McSorley ST, Steele CW, McMahon AJ (2018) Meta-analysis of oral antibiotics, in combination with preoperative intravenous antibiotics and mechanical bowel preparation the day before surgery, compared with intravenous antibiotics and mechanical bowel preparation alone to reduce surgical-site infections in elective colorectal surgery. BJS open 2(4):185-194. https://doi.org/10.1002/bjs5.68

25. Mulder T, Crolla R, Kluytmans-van den Bergh MFQ, van Mourik MSM, Romme J, van der Schelling GP, Kluytmans J (2019) Preoperative oral antibiotic prophylaxis reduces surgical site infections after elective colorectal surgery: results from a before-after study. Clin Infect Dis 69(1):93-99. https://doi.org/10.1093/cid/ ciy 839

26. Hata H, Yamaguchi T, Hasegawa S, Nomura A, Hida K, Nishitai R, Yamanokuchi S, Yamanaka T, Sakai Y (2016) Oral and parenteral versus parenteral antibiotic prophylaxis in elective laparoscopic colorectal surgery (JMTO PREV 07-01): a phase 3, multicenter, openlabel, randomized trial. Ann Surg 263(6):1085-1091. https://doi. org/10.1097/sla.0000000000001581

27. Kaiser AB, Herrington JL Jr, Jacobs JK, Mulherin JL Jr, Roach AC, Sawyers JL (1983) Cefoxitin versus erythromycin, neomycin, and cefazolin in colorectal operations. Importance of the duration of the surgical procedure. Ann Surg 198(4):525-530. https://doi.org/10. 1097/00000658-198310000-00012

28. Lewis RT (2002) Oral versus systemic antibiotic prophylaxis in elective colon surgery: a randomized study and meta-analysis send a message from the 1990s. Can J Surg 45(3):173-180

29. Múñez E, Ramos A, Espejo T, Vaqué J, Sánchez-Payá J, Pastor V, Asensio A (2011) Microbiology of surgical site infections in abdominal tract surgery patients. Cir Esp 89(9):606-612. https://doi. org/10.1016/j.ciresp.2011.05.008

30. Abis GSA, Stockmann H, Bonjer HJ, van Veenendaal N, van Doorn-Schepens MLM, Budding AE, Wilschut JA, van Egmond M, Oosterling SJ (2019) Randomized clinical trial of selective decontamination of the digestive tract in elective colorectal cancer surgery (SELECT trial). Br J Surg 106(4):355-363. https://doi. org/10.1002/bjs.11117

31. Mulder T, Kluytmans-van den Bergh M, Vlaminckx B, Roos D, de Smet AM, de Vos tot Nederveen Cappel R, Verheijen P, Brandt A, Smits A, van der Vorm E, Bathoorn E, van Etten B, Veenemans J, Weersink A, Vos M, van 't Veer N, Nikolakopoulos S, Bonten M, Kluytmans J (2020) Prevention of severe infectious complications after colorectal surgery using oral non-absorbable antimicrobial prophylaxis: results of a multicenter randomized placebocontrolled clinical trial. Antimicrob Resist Infect Control 9(1):84. https://doi.org/10.1186/s13756-020-00745-2

32. Musson DG, Majumdar A, Holland S, Birk K, Xi L, Mistry G, Sciberras D, Muckow J, Deutsch P, Rogers JD (2004) Pharmacokinetics of total and unbound ertapenem in healthy elderly subjects. Antimicrob Agents Chemother 48(2):521-524. https:// doi.org/10.1128/aac.48.2.521-524.2004 
33. The European Committee on Antimicrobial Susceptibility and Testing. Breakpoint tables for interpretation of MICs and zone diameters. version 11.0. 2021. http://www.eucast.org. Accessed 06. 04.2021.

34. Deierhoi RJ, Dawes LG, Vick C, Itani KMF, Hawn MT (2013) Choice of intravenous antibiotic prophylaxis for colorectal surgery does matter. J Am Coll Surg 217(5):763-769. https://doi.org/10. 1016/j.jamcollsurg.2013.07.003

35. Olivas AD, Shogan BD, Valuckaite V, Zaborin A, Belogortseva N, Musch M, Meyer F, Trimble WL, An G, Gilbert J, Zaborina O, Alverdy JC (2012) Intestinal tissues induce an SNP mutation in Pseudomonas aeruginosa that enhances its virulence: possible role in anastomotic leak. PLoS One 7(8):e44326. https://doi.org/10. 1371/journal.pone.0044326

36. Shogan BD, Belogortseva N, Luong PM, Zaborin A, Lax S, Bethel C, Ward M, Muldoon JP, Singer M, An G, Umanskiy K, Konda V, Shakhsheer B, Luo J, Klabbers R, Hancock LE, Gilbert J, Zaborina O, Alverdy JC (2015) Collagen degradation and MMP9 activation by Enterococcus faecalis contribute to intestinal anastomotic leak. Sci Trans1 Med. 7(286):286-268. https://doi.org/10.1126/ scitranslmed.3010658
37. Schardey HM, Wirth U, Strauss T, Kasparek MS, Schneider D, Jauch KW (2020) Prevention of anastomotic leak in rectal cancer surgery with local antibiotic decontamination: a prospective, randomized, double-blind, placebo-controlled single center trial. Int J Color Dis 35(5):847-857. https://doi.org/10.1007/s00384-02003544-8

38. Papp G, Saftics G, Szabo BE, Baracs J, Vereczkei A, Kollar D, Olah A, Meszaros P, Duboczki Z, Bursics A (2021) Systemic versus Oral and Systemic Antibiotic Prophylaxis (SOAP) study in colorectal surgery: prospective randomized multicentre trial. Br J Surg 108:271-276. https://doi.org/10.1093/bjs/znaa131

39. Koskenvuo L, Lehtonen T, Koskensalo S, Rasilainen S, Klintrup K, Ehrlich A, Pinta T, Scheinin T, Sallinen V (2019) Mechanical and oral antibiotic bowel preparation versus no bowel preparation for elective colectomy (MOBILE): a multicentre, randomised, parallel, single-blinded trial. Lancet. 394(10201):840-848. https://doi.org/ 10.1016/s0140-6736(19)31269-3

Publisher's note Springer Nature remains neutral with regard to jurisdictional claims in published maps and institutional affiliations. 